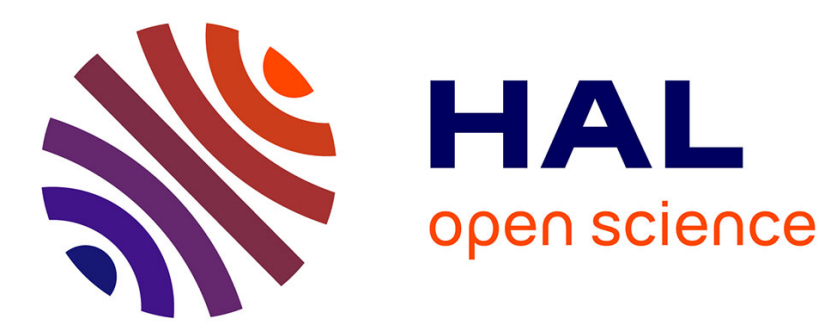

\title{
Investigation of porosity and permeability effects from microstructure changes during limestone dissolution
}

Catherine Noiriel, Philippe Gouze, Dominique Bernard

\section{To cite this version:}

Catherine Noiriel, Philippe Gouze, Dominique Bernard. Investigation of porosity and permeability effects from microstructure changes during limestone dissolution. Geophysical Research Letters, 2004, 31 (23), L24603 (4 p.). 10.1029/2004GL021572 . hal-00350625

\section{HAL Id: hal-00350625 https://hal.science/hal-00350625}

Submitted on 1 Feb 2021

HAL is a multi-disciplinary open access archive for the deposit and dissemination of scientific research documents, whether they are published or not. The documents may come from teaching and research institutions in France or abroad, or from public or private research centers.
L'archive ouverte pluridisciplinaire HAL, est destinée au dépôt et à la diffusion de documents scientifiques de niveau recherche, publiés ou non, émanant des établissements d'enseignement et de recherche français ou étrangers, des laboratoires publics ou privés. 


\title{
Investigation of porosity and permeability effects from microstructure changes during limestone dissolution
}

\author{
Catherine Noiriel, ${ }^{1,2}$ Philippe Gouze, ${ }^{1}$ and Dominique Bernard ${ }^{3}$ \\ Received 21 September 2004; revised 29 October 2004; accepted 17 November 2004; published 21 December 2004.
}

[1] We studied experimentally the dissolution of a porous limestone core during $\mathrm{CO}_{2}$-enriched water injection. We measured the changes in porosity and permeability arising from modifications of the pore network geometry and the fluid-rock interface. A methodology based on periodic X-ray microtomography imaging was implemented to record the evolution of the time- and scale-dependent microstructures with a spatial resolution of $4.91 \mu \mathrm{m}$. Two processes were successively involved in the rapid permeability increase of the sample, as documented from microscale to core-scale measurements. First, the microcrystalline phase was partially dissolved, associated with displacement of mineral particles. During this process, the exponent $n$ of the power law $k \sim \phi^{n}$ decreased continuously. Secondly the sparitic phase dissolved, accompanied by a decrease of the pore wall roughness and an increase of the pore connectivity. This second period was characterized by a constant value of $n$. The reactive surface decreased noticeably during the transition from the microcrystalline to the sparitic dissolution periods, whereas the effective porosity increased strongly. INDEX TERMS: 1010 Geochemistry: Chemical evolution; 5114 Physical Properties of Rocks: Permeability and porosity; 5112 Physical Properties of Rocks: Microstructure; 5139 Physical Properties of Rocks: Transport properties; 5194 Physical Properties of Rocks: Instruments and techniques. Citation: Noiriel, C., P. Gouze, and D. Bernard (2004), Investigation of porosity and permeability effects from microstructure changes during limestone dissolution, Geophys. Res. Lett., 31, L24603, doi:10.1029/2004GL021572.

\section{Introduction}

[2] $\mathrm{CO}_{2}$ injection is used routinely in the petroleum industry to enhance oil recovery. It has been proposed to apply this method to underground carbon sequestration for mitigating $\mathrm{CO}_{2}$ concentration increase in the atmosphere. Depleted oil reservoirs and large confined aquifers are potential geologic host formations. In those environments, carbonates are often present either as the main minerals or as the cement fraction in sandstones. Carbonate mass transfers are rapid processes when compared to silicate alteration. It is therefore the major chemical process affecting the properties of a reservoir in the course of an injection of $\mathrm{CO}_{2}$. In the vicinity of the injection well, permeability changes will control the injection capacity. Conversely, modifications of

\footnotetext{
${ }^{1}$ Laboratoire de Tectonophysique, ISTEEM, CNRS-Université Montpellier II, Montpellier, France.

${ }^{2}$ Also at Ecole des Mines de Paris, CIG, Fontainebleau, France.

${ }^{3}$ Institut de Chimie de la Matière Condensée de Bordeaux, CNRS, Pessac, France.
}

Copyright 2004 by the American Geophysical Union. 0094-8276/04/2004GL021572 the porosity and of the surface area of the fluid-rock interface will severely constrain the capacity of the reservoir to buffer the fluid acidity and to store carbon in mineral. The heterogeneity of the pore structure, in relation with the fluid velocity fluctuations, will determine the configuration of the interface between the $\mathrm{CO}_{2}$ gas phase and the resident fluid, and therefore, will control the rate of dissolution of the $\mathrm{CO}_{2}$ in the fluid. Reliable predictions of those property changes as a function of time and space are necessary for setting up effective $\mathrm{CO}_{2}$ injection procedures.

[3] The relationship between connected porosity and permeability is complex and highly variable according to the rock structure, mineralogy, and geological process involved. The most commonly used models relating permeability to porosity in porous media are based on the original work of Carman [1937]. Permeability $k$ and effective porosity $\phi_{e}$ (i.e., pore space that effectively contributes to fluid flow) are linked through a power-law relationship, $k \propto \phi_{e}^{n}$ or, alternatively, a linear combination of power-laws $k \propto \sum_{i} a_{i} \phi_{e}^{n_{i}}$ [Pape et al., 1999]. Asymptotically, it can be considered that the exponent $n$ varies continuously with the evolution of the porosity versus permeability, so that $n$ is the slope of the tangent at any point of the $\log (k)$ versus $\log \left(\phi_{e}\right)$ curve. Yet, experimental evidence indicates that a given value of $n$ is generally valid over a finite $\phi_{e}$ range [Bourbié and Zinszner, 1985]. Conversely, variable values of the $n$ exponent indicate spatial heterogeneity in the evolution of the pore structure or the sequential activation of distinctly different processes. However, the predictive capacity of a model is poor if $n$ is not a function of $\phi_{e}$ alone or rather of a restricted number of independent measurable parameters.

[4] The aim of this letter is to document the parameters controlling the dynamics of the permeability versus porosity relationship based on the characterisation of the microstructural changes during dissolution of a natural porous limestone by $\mathrm{CO}_{2}$-enriched water. Our experimental methodology combines periodic X-ray microtomography imaging and fluid chemical analysis, and continuous measurement of the sample-scale permeability. We propose a quantification with time of the modifications of the principal parameters characterizing the hydrodynamic properties, the fluid-rock interface structure and the kinetics of the chemical reactions.

\section{Experimental Method and Results}

\subsection{Experiment}

[5] A sample of middle Oxfordian crinoïdal limestone was extracted from a quarry in the Lérouville formation (Paris Basin). The rock is essentially composed of millimeter-size sparitic re-crystallised echinoderm fragments of calcite. Pores are partially filled with poorly cohesive secondary microcrystals of calcite and altered material $(\sim 5 \%$ of the solid phase). 
Table 1. Characteristics of the 3 Stages of Dissolution

\begin{tabular}{lccc}
\hline & $t_{0} \leq t \leq t_{1}$ & $t_{1}<t \leq t_{2}$ & $t_{2}<t \leq t_{3}$ \\
\hline Flow rate $Q\left(\mathrm{~cm}^{3} \cdot \mathrm{h}^{-1}\right)$ & 300 & 300 & 100 \\
Duration $t(\mathrm{~h})$ & 1.4 & 12.5 & 8.5 \\
pH inlet & $4.7 \pm 0.1$ & $3.9 \pm 0.1$ & $3.8 \pm 0.1$ \\
pH outlet & $5.6 \pm 0.2$ & $5.6 \pm 0.2$ & $5.2 \pm 0.1$ \\
Average $\Delta_{\mathrm{Ca}}\left(\mathrm{mol}^{-1}\right)$ & $0.3210^{-3} \pm 0.1010^{-3}$ & $0.4910^{-3} \pm 0.0510^{-3}$ & $1.0710^{-3} \pm 0.0710^{-3}$ \\
\hline
\end{tabular}

[6] The experiment consisted in injecting $\mathrm{CO}_{2}$-enriched water through a cylindrical epoxy-resin-coated core of $9 \mathrm{~mm}$ diameter $(D)$ and $21 \mathrm{~mm}$ length $(L)$. The experiment was conducted at room temperature using three types of fluids successively: $F^{\left(t_{0}-t_{1}\right)}$ was water equilibrated with limestone at atmospheric partial pressure of $\mathrm{CO}_{2}, F^{\left(t_{1}-t_{2}\right)}$ deionized water, and $F^{\left(t_{2}-t_{3}\right)}$ a $10^{-2}$ molar solution of $\mathrm{NaCl}$ prepared from reagent-grade salt dissolved in deionized water. Each of these fluids was used during a given flow-through stage after being equilibrated with $\mathrm{CO}_{2}$ at the partial pressure of $0.10 \pm 0.01 \mathrm{MPa}$. The flow-trough stage $i$ had a duration $\Delta t=t_{i+1}-t_{i}$ given in Table 1 with constant flow rate $Q_{i}$ and inlet fluid composition $\mathrm{F}^{\left(\mathrm{t}_{\mathrm{i}}-\mathrm{t}_{\mathrm{i}-1}\right)}$. The sample was imaged using computed microtomography (CMT) before the experiment $\left(t=t_{0}\right)$ and at the end of each flow-through stage $(t=$ $t_{1}, t_{2}$ and $\left.t_{3}\right)$. A review of CMT is given by Mees et al. [2003]. Here, the ID19 X-ray beam of the European Synchrotron Radiation Facility was used. This apparatus allows imaging the rock structure over four spatial scales with a pixel size of $4.91 \mu \mathrm{m}$ using a $2048^{2}$ pixels camera. Inlet/outlet differential pressure and $\mathrm{pH}$ were monitored. The solution was sampled for calcium content and analyzed by ICP-AES. Macroscale changes in permeability $k(t)$ were calculated from the pressure drop $\Delta P(t)$ according to Darcy's law. As the core is composed almost completely of calcite, the macroscopic (sample-scale) porosity $\phi^{*}(t)$ accessible to the dissolution reaction can be calculated from the measured calcium balance:

$$
\phi^{*}(t)=\phi_{0}^{*}+\frac{4 v}{\pi D^{2} L} \int_{\tau=o}^{\tau=t} r(\tau) d \tau
$$

where $\phi_{0}^{*}$ denotes the accessible porosity at $t_{0}, v$ is the calcite molar volume and $r$ is the macroscopic rate of dissolution $\left(\right.$ mol.s $\left.^{-1}\right) ; r \approx Q \Delta_{\mathrm{Ca}}$, with $\Delta_{\mathrm{Ca}}$ the difference in calcium concentration in the fluid between the output and the input of the core. Alternatively, another estimate of the total porosity, $\phi_{T}(t)$, can be calculated from the CMT data by discriminating the voids from the solid phase. The measured core permeability and the two porosity estimations are reported in Figure 1. Porosity increased linearly with time whereas permeability increased as a power law of time.

\subsection{Rate of Dissolution and Reactive Surface}

[7] Differences in Ca concentration and $\mathrm{pH}$ between core outlet and inlet can be directly related to the progress of calcite dissolution reaction and to the fluid replacement in the core. The macroscopic dissolution rate of the rock sample is $r=\int \kappa(1-\Omega)^{n} \mathrm{~d} A$, where, $\kappa$ is the kinetic rate constant, $\Omega$ is the calcite saturation index and $A_{s}$ is the area of the fluid-calcite interface [Lasaga, 1981]. In our experiment, the value of $(1-\Omega)^{n} \approx 1$ because of the high level of disequilibrium imposed by the constant injection of $\mathrm{CO}_{2}$-rich fluid $\left(\Omega \approx 10^{-3}\right)$. Far from equilibrium, $\kappa=$ $\kappa_{1} a_{H}+\kappa_{2} a_{H_{2} \mathrm{CO}_{3}}+\kappa_{3} a_{\mathrm{H}_{2} \mathrm{O}}$, where $a_{i}$ denotes the activity of species $i$, and $\kappa_{1}, \kappa_{2}$ and $\kappa_{3}$ are the kinetic rate constants at $25^{\circ} \mathrm{C}\left(\right.$ mol. $\left.\mathrm{m}^{-2} \cdot \mathrm{s}^{-1}\right)$ [Plummer et al., 1978]. For acidic fluids (i.e., $\mathrm{pH}<4.5$ ), the rate of dissolution is principally $\mathrm{pH}$ dependent and therefore $r$ decreases progressively from the inlet to the outlet side as $\mathrm{H}^{+}$ions are consumed during the calcite dissolution reaction. We indeed observed a slight localization of the dissolution at the sample inlet. The macroscopic dissolution rate is then:

$$
r \approx \kappa_{1} \int_{A_{s}} a_{H^{+}} \mathrm{d} A=\kappa_{1} A_{s}\left\langle a_{H^{+}}\right\rangle_{A_{s}}
$$

where $\left\langle a_{H^{+}}\right\rangle_{A_{s}}$ denotes the activity of $\mathrm{H}^{+}$averaged over the fluid-rock surface.

[8] A basic concept in chemical kinetics is that the overall mass transfer of a given species results from a set of serial chemical and physical processes, including (1) the transport of the reactants towards the surface, (2) the adsorption of the reactants at the reaction site, (3) the heterogeneous reactions, (4) the desorption of the products away from the reaction site, and (5) the transport of the products away from the reactive surface to the solution. The overall rate of dissolution is controlled by the slowest process. All those processes will affect the local values of the $\mathrm{H}^{+}$activity and the value of its surface average. Because the only available data related to $\mathrm{H}^{+}$activity are the $\mathrm{pH}$ measured at the inlet and at the outlet of the core, we have to assume that the sample-scale averaged value of the dissolution rate is $r \approx \kappa_{1} A_{S}\left\langle a_{H^{+}}\right\rangle_{A_{s}}=$ $\kappa_{1} A_{r}\left\langle a_{H^{+}}\right\rangle$, where $\left\langle a_{H^{+}}\right\rangle$is the mean $\mathrm{H}^{+}$activity in the core fluid and $A_{r}$ denotes the area of effective reactive surface. This effective surface of reaction can be calculated from the sample-scale data by the relation $Q \Delta_{\mathrm{Ca}} \approx$ $A_{r} \kappa_{1}\left\langle a_{H^{+}}\right\rangle$. The effective reactive surface decreased from $7.310^{-3} \mathrm{~m}^{2}$ during the flow-trough stage 1 to $4.510^{-3} \mathrm{~m}^{2}$

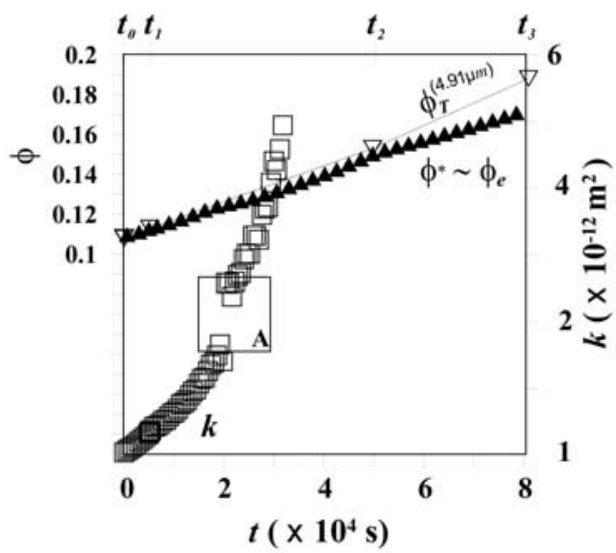

Figure 1. Total porosity $\phi_{T}$, accessible porosity $\phi^{*}$ and permeability $k$ versus time. Zone denoted A indicates a discontinuity in the pressure measurement. 


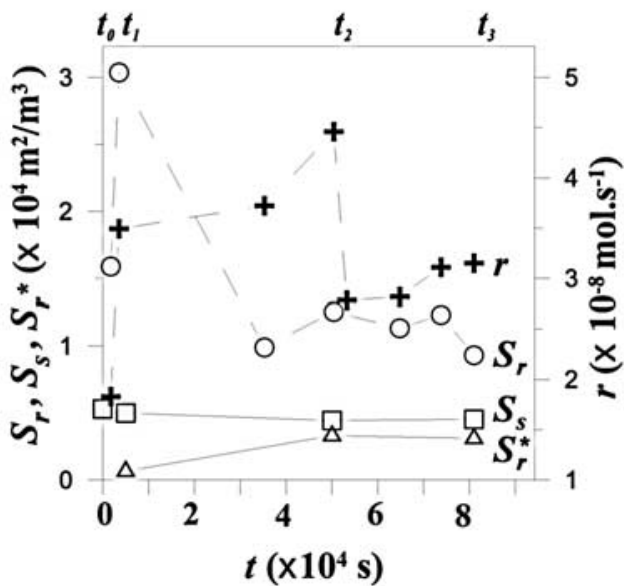

Figure 2. Dissolution rate $r$, reactive surface $S_{r}$, specific surface $S_{s}$, and surface of dissolution $S_{r}^{*}$, versus time. The connecting lines are only used to guide the eyes.

and $1.810^{-3} \mathrm{~m}^{2}$ during stages 2 and 3 , respectively. During stage 1, the large values of $A_{r}$ can be related to the presence of the microcrystalline calcite which present higher reactive surface than sparitic calcite. As microcrystalline calcite was rapidly dissolved, $A_{r}$ decreased to lower values. The evolutions of $r$ and of $S_{r}=4 A_{r} / \pi \mathrm{D}^{2} \mathrm{~L}$ are given in Figure 2 .

\subsection{Microstructure Analysis}

[9] Determining space and time modifications of the microstructure requires locating the fluid rock interface of $V^{\left(t_{0}\right)}$ to $V^{\left(t_{3}\right)}$ in a common coordinate system. The transformation parameters permitting this change of reference frame were determined for a sub-domain of about $400^{3}$ voxels (or $\sim 8 \mathrm{~mm}^{3}$ ). We obtained four images of the sub-volume, noted $v^{\left(t_{0}\right)}$ to $v^{\left(t_{3}\right)}$. After binarization of the images into solid and void, the changes with time of each voxel could be measured. The history of $v^{\left(t_{0}\right)}$ to $v^{\left(t_{3}\right)}$ is stored in a four-bits encoded matrix $\mathbf{M}_{v}[b(x, y, z)]$ using the following algorithm: at time $t=i$ and for each voxel, the bit number $j(j=1 \ldots 4)$ of the matrix element $b$ is set to 1 if the voxel belongs to the solid phase and 0 otherwise. The final value assigned to a voxel $b(x, y, z)$ summarizes its evolution. The histogram of the obtained values shows that 6 separate scenarios represent $99.4 \%$ of the occurrences (Table 2). Then, $\mathbf{M}_{v}$ can be partitioned in six regions and it becomes easy to quantify and localize the volume of rock dissolved or displaced in the course of experiment (Figure 3). The specific surface $S_{S}$ and the effective surface of reaction $S_{r}^{*}$ are obtained by dividing the area of the total fluid-rock interface $\left(A_{s}^{\left(t_{0}\right)} \ldots A_{s}^{\left(t_{3}\right)}\right)$ and of the fluid-rock interface modified during the experiment $A_{r}^{*\left(t_{0}-t_{1}\right)} \ldots A_{r}^{*\left(t_{2}-t_{3}\right)}$, respectively, by the

Table 2. The 6 Principal Scenarios Identified From the CMT Registration Procedure and Their \%-Occurrence During the Experiment

\begin{tabular}{ll}
\hline$\%$ & \multicolumn{1}{c}{ Scenario } \\
\hline 58.3 & "Solid phase" voxels from $t_{0}$ to $t_{3}$ \\
26.5 & "Pore volume" voxels from $t_{0}$ to $t_{3}$ \\
1.7 & "Solid phase" voxels dissolved or displaced for $t \leq t_{1}$ \\
1.1 & "Pore volume" voxels replaced by a moving particle for $t \leq t_{1}$ \\
7.2 & and then dissolved for $t>t_{1}$ \\
4.6 & "Solid phase" voxels dissolved for $t_{1}<t \leq t_{2}$ \\
\hline
\end{tabular}

volume of the sub-domain (Figure 2). At the end of the first flow-through stage $\left(t_{1}\right)$, the changes in the area of fluidrock interface were small ( $\sim 13 \%$ of the total interface area), but important displacements of particles were observed (Figure 3) and interpreted as the result of progressive crumbling of the microcrystalline phase during its dissolution. During the stage 2 and 3, sparitic-calcite recrystallized crinoids were dissolved; dissolution occurred on $76 \%$ and $69 \%$, respectively, of the total fluid-rock interface.

\section{Discussion}

\subsection{Porosity and Fluid-Rock Interface Changes}

[10] The value of the macroscopic accessible porosity at $t=0$ is unknown, therefore equation (1) is initialized from the CMT measurement of the total porosity: $\phi_{0}^{*}=\phi_{T}(t=0)$. Consequently, comparing the rate of increase of the accessible porosity to that of the total porosity is meaningful whereas value-to-value comparison is meaningless. Figure 1 shows that the evolutions of $\phi^{*}$ and $\phi_{T}$ were similar between $t_{0}$ and $t_{2}$, but diverged during the last flow-through stage for which the flow rate was reduced from 300 to $100 \mathrm{~cm}^{3} \mathrm{~h}^{-1}$. The similarity between $\phi^{*}$ and $\phi_{T}$ evolution up to $t_{2}$ indicates that the fluid composition was homogeneous along the core length. At high flow rate, the dissolution gradient across the core length was small and the averaged composition of the fluid was representative of the average dissolution process. Conversely, for $t>t_{2}$, one can attribute the dissimilarity between $\phi^{*}$ and $\phi_{T}$ evolution to the increasing localization of the dissolution at the inlet side of the core. As a result, the gradient of composition along the core length axis must have been significant and the core-averaged value of the reaction rate cannot characterize precisely the dissolution process.

[11] In their review paper, Bernabé et al. [2003] propose to define the effective porosity $\phi_{e}$ as the total porosity minus the fraction of the porosity where the fluid velocity is very

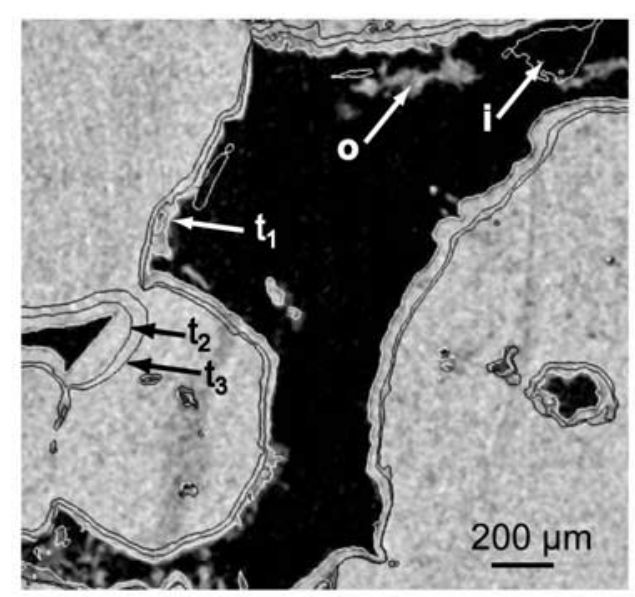

Figure 3. CMT cross section of the core. At $t_{0}$, black and grey colors denote the pore void and solid, respectively. The position of the fluid-rock interface is indicated by the white line for $t_{1}$, and by the concentric black lines for $t_{2}$ and $t_{3}$. The displacement of suspended particles at $t_{1}$ is exemplified here by the disappearance of the particle (denoted o) and the arrival of the particle denoted $i$. The changes with time in the fluid-rock interface roughness can be easily observed. 


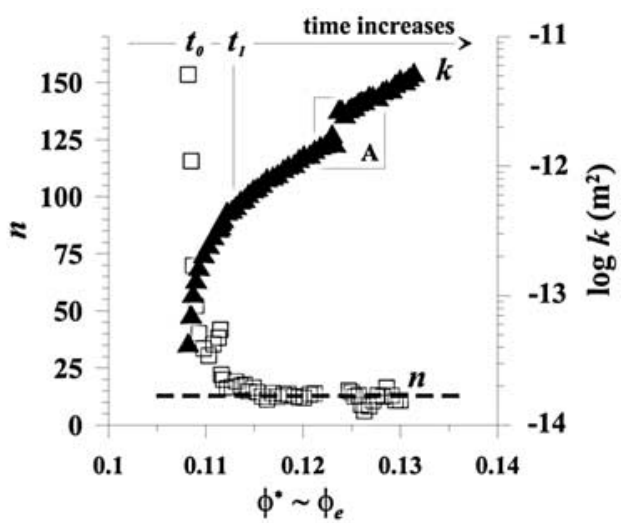

Figure 4. Permeability (log axis) and power law exponent $n$ versus porosity.

low or zero, such as dead ends, unconnected pores and microporosity forming the pore roughness. According to this definition, effective porosity is not calculable unless the complete transient 3D flow field is known. Nevertheless, as the dissolution rate is controlled by local velocity in the vicinity of the fluid-rock interface, $\phi^{*}$ (equation (1)) theoretically represents a useful evaluation of the effective porosity $\phi_{e}$. However, because of the indetermination of the initial $\left(t=t_{0}\right)$ value of $\phi^{*}$, only the proportionality $\phi_{e} \sim \phi^{*}$ and the equality $\partial \phi^{*} / \partial t=\partial \phi_{e} / \partial t$ are usable.

[12] The evolution of the fluid-rock interface is also a valuable indicator of the active processes. The specific surface of a digitalized image of a smooth-surfaced porous media, such as sphere assemblage, is larger than the actual surface. Conversely, for natural porous medium the digitalized specific surface is generally smaller than the actual because of the roughness of the fluid-rock interface at length scale smaller than the imaging pixel size. Subsequently, the ratio of $S_{r}$ (measured from the fluid chemistry) on $S_{r}^{*}$ (obtained from digitalized images) can be used to evaluate the change in the roughness of the fluid-rock interface at length scale smaller than the imaging resolution. Examination of Figures 2 and 3 shows that fluid-rock interface roughness decreased strongly during the dissolution of the microcrystalline phase. Then, for $t \geq 310^{4} \mathrm{~s}$, the rate of decrease of roughness slowed down as all the microcrystalline phase had been dissolved. It is interesting to note that this behaviour is correlated with the evolution of the exponent $n$ characterizing the relation between $k$ and $\phi$ (see section 3.2).

\subsection{Porosity Versus Permeability Relationship}

[13] Figure 4 displays the evolution of permeability versus porosity during the experiment. Permeability increased rapidly by one order of magnitude in the early minutes of the experiment whereas porosity remained almost unchanged over the same interval of time. At this stage, the microcrystalline phase dissolved rapidly because of the large value of the reactive surface (see section 2.2); this induced a rapid increase of the pore structure connectivity. The power law exponent $n$ decreased rapidly (one order of magnitude). Then, as the microcrystalline phase was completely dissolved, the value of $n$ stabilized at about 13, characterizing the dissolution of the spariterecrystallized crinoids. Similarly high values of the power law exponent were previously reported by other authors studying precipitation processes [e.g., Xu et al., 2004]. McCune et al. [1979] described similar results during the percolation of acidic fluids in limestone and sandstone. The authors attributed the high values of $n$ to the displacement of fine mobile rock particles in the pore network, similarly to what is observed in the first stage of our experiment. When all the accessible fraction of the microcrystalline phase was dissolved (including remobilized particles), $n=13$ corresponds to a decrease of the pore roughness as observed on the CMT images difference $t^{2}-t^{1}$ (Figure 3). Finally, for $t>t_{2}$, the chemical erosion of the sparitic grains became nearly homogeneous, i.e., pore roughness changes were small. The increase of pore connectivity is also observed in the images and one may expect that the $n$ exponent should then reach another value. However, this hypothesis cannot be proved by quantitative results because permeability recording stopped at $t=9$ hours as $k$ reached $510^{-12} \mathrm{~m}^{2}$, the maximum value being measurable by the differential pressure gauge. Incidentally, note that the influence of the initial fluid composition on the $k-\phi$ relationship is not detectable, indicating that this parameter is probably minor for high $\mathrm{CO}_{2}$ partial pressure environments.

\subsection{Concluding Remarks}

[14] Combining imaging method and chemical analysis allowed us to assess simultaneously the changes in effective porosity, total porosity, reactive and specific surfaces while measuring permeability during the flow-through experiment. Using these parameters it is now possible to document the $k-\phi_{e}$ evolution and to relate it to the structural modifications of the pore space. This information is essential to constrain reservoir modeling for large-scale injection of $\mathrm{CO}_{2}$. Indeed, significant and rapid modifications of the hydrodynamic properties are expected, which will impact the injection procedure and the trapping efficiency of $\mathrm{CO}_{2}$.

\section{References}

Bernabé, Y., U. Mok, and B. Evans (2003), Permeability-porosity relationships in rocks subjected to various evolution processes, Pure Appl. Geophys., 160, 937-960

Bourbié, T., and B. Zinszner (1985), Hydraulic and acoustic properties as function of porosity in Fontainebleau sandstone, J. Geophys. Res., 90, $11,524-11,532$.

Carman, P. C. (1937), Fluid flow through granular beds, Trans. Inst. Chem. Eng., 15, 150-166.

Lasaga, A. C. (1981), Transition state theory, in Kinetics of Geochemical Processes, Rev. Mineral., vol. 8, edited by A. C. Lasaga and R. J. Kirkpatrick, 135-169, Mineral. Soc. of Am., Washington, D. C.

McCune, C. C., H. S. Fogler, and W. E. Kline (1979), An experimental technique for obtaining permeability-porosity relationships in acidized porous media, Ind. Eng. Chem. Fundam., 18, 188-191.

Mees, F., R. Swennen, M. Van Geet, and P. Jacobs (Eds.) (2003), Applications of X-ray computed tomography in geosciences, Geol. Soc. London Spec. Publ., 215, $234 \mathrm{p}$

Pape, H., C. Clauser, and J. Iffland (1999), Permeability prediction based on fractal pore-space geometry, Geophysics, 64, 1447-1460.

Plummer, L. N., T. M. L. Wigley, and D. L. Parkhurst (1978), The kinetics of calcite dissolution in $\mathrm{CO}_{2}$-water systems at $5^{\circ}$ to $60^{\circ} \mathrm{C}$ and 0.0 to $1.0 \mathrm{~atm} \mathrm{CO}_{2}, \mathrm{Am}$. J. Sci., 278, 179-216.

Xu, T., Y. Ontoy, P. Molling, N. Spycher, M. Parini, and K. Pruess (2004), Reactive transport modeling of injection well scaling and acidizing at Tiwi Field, Philippines, Geothermics, 33, 477-491.

D. Bernard, Institut de Chimie de la Matière Condensée de Bordeaux, CNRS, F-33608 Pessac Cedex, France.

P. Gouze and C. Noiriel, Laboratoire de Tectonophysique, ISTEEM, CNRS-Université Montpellier, F-34095 Montpellier Cedex 5, France. (noiriel@msem.univ-montp2.fr) 